\title{
Comparação entre o modelo de malha móvel e o modelo de fonte de quantidade de movimento para a simulação numérica do princípio de funcionamento de tanques misturadores
}

Comparison between the sliding mesh model and the momentum source model for the numerical simulation of the working principle of mixing tanks

\author{
L. L. Soares ${ }^{1 *}$; M. M. Galarça ${ }^{1,2}$; E. D. dos Santos ${ }^{1} ;$ J. A. A. Oliveira Júnior ${ }^{3}$ \\ IPrograma de Pós-Graduação em Modelagem Computacional, Universidade Federal do Rio Grande, 96203-900, \\ Rio Grande-RS, Brasil \\ ${ }^{2}$ Engenharia mecânica, Instituto Federal do Rio Grande do Sul, 96201-460, Rio Grande-RS, Brasil \\ ${ }^{3}$ ESSS Ltda, 88032-700, Florianópolis-SC, Brasil.
}

*laisaluiz.ls@gmail.com

\begin{abstract}
O presente trabalho tem o intuito de apresentar uma comparação entre dois modelos numéricos capazes de simular o princípio de funcionamento de tanques misturadores, os quais são utilizados em diversas indústrias químicas, entre elas, para a produção de biodiesel. O objetivo é realizar uma comparação entre os resultados obtidos com estes modelos e os resultados encontrados na literatura, a fim de mostrar a capacidade de predição dos modelos desenvolvidos e o esforço computacional de cada um deles. As simulações numéricas foram realizadas utilizando o software FLUENT, baseado no Método de Volumes Finitos (MVF). A comparação dos resultados entre o presente trabalho e dados encontrados na literatura, foi realizada a partir da previsão dos campos de velocidade monitorados no escoamento. Em geral, foi encontrado bom acordo entre os resultados do presente trabalho e resultados da literatura, com relação ao comportamento do escoamento, em determinados pontos que foram analisados no interior do tanque. Foi atestado também, uma grande diferença no tempo e esforço computacional entre os dois modelos numéricos.
\end{abstract}

Palavras-chave: Simulação numérica, tanque misturador, modelo numérico.

In this paper the aims to present a comparison between two numerical models capable of simulating the working principle of mixing tanks, which are used in several chemical industries, among them, for the production of biodiesel. The objective is to make a comparison between the results obtained with these models and the results found in the literature, in order to show the predictive capacity of the models developed and the computational effort of each of them. The numerical simulations were performed using FLUENT software, based on the Finite Volume Method (FVM). The comparison of the results between the present paper and the data found in the literature was performed from the prediction of the velocity fields monitored in the flow. In general, a good agreement was found between the results of the present paper and the literature results, in relation to the flow behavior, at certain points that were analyzed inside the tank. It was also certified a large difference in time and computational effort between the two numerical models.

Keywords: Numerical simulation, stirred tank, numerical model.

\section{INTRODUÇÃO}

Os tanques misturadores são bastante utilizados em diversos processos químicos onde a agitação e a mistura de reagentes são necessários para a obtenção do produto final, podendo ser utilizados para misturas de única ou múltiplas fases. Indústrias como a alimentícia, de fármacos e de combustíveis, são exemplos da utilização destes tanques de mistura. Em geral, a configuração geométrica destes tanques consiste de um vaso cilíndrico, composto de um ou mais impulsores (dependendo da necessidade de mistura) localizados no centro do vaso e com uma determinada distância do fundo. Em alguns casos, os tanques também podem possuir anteparos localizados nas paredes do vaso com igual espaçamento entre eles, afim de aumentar a turbulência do escoamento. A Figura 1, mostra um domínio computacional que representa uma configuração usual de um tanque misturador. 
Segundo Patwardhan (2001) [1] e Xu \& Mcgrath (1996) [2] a qualidade de mistura é altamente dependente da hidrodinâmica que ocorre no interior do tanque, isto é, do campo de escoamento gerado pelo impulsor. Além disso, o número de impulsores e suas localizações, tamanho das lâminas, bem como a configuração do tanque (número e forma de anteparos, diâmetro do tanque e nível de líquido) também têm grande impacto no campo de escoamento. Com isso, o escoamento pode ter diferentes comportamentos, mas em geral ele é altamente turbulento, sendo que, a turbulência é a responsável pelo transporte dispersivo de quantidade de movimento, calor e massa [1].

Segundo Mahmud et al. (2006) [3] para que se possa aumentar a qualidade do produto final, principalmente quando ocorrem reações químicas, é necessário a obtenção de alguns conhecimentos da influência da hidrodinâmica na mistura dos reagentes. Desta forma, os modelos numéricos baseados na mecânica dos fluidos computacional (CFD) auxiliam no entendimento detalhado do escoamento turbulento no interior de tanques misturadores, com um custo relativamente baixo se comparado ao experimental. Por outro lado, a acurácia dos resultados se torna altamente dependente da forma como será tratada a interação entre fluido e fronteira física, da malha computacional, dos esquemas de discretização para o transporte dos termos convectivos e dos modelos físicos que representam o transporte turbulento. Neste sentido, para similar numericamente o princípio de funcionamento de um tanque misturador, é necessário que o modelo seja capaz de simular, da melhor forma possível, a interação entre impulsor e fluido. Assim, no presente trabalho, dois tipos de modelos numéricos são aplicados e comparados entre eles: o modelo de malha móvel e o modelo de fonte de quantidade de movimento. Este último será chamado de modelo de termo fonte a partir daqui.

Alguns trabalhos com simulação numérica envolvendo a utilização destes dois modelos numéricos (malha móvel e termo fonte), vêm sendo desenvolvidos com o objetivo de estudar, basicamente, o comportamento do escoamento no interior de tanques misturadores. Entre estes trabalhos, podem ser citados por exemplo, o trabalho de Xu \& Mcgrath (1996) [2] que desenvolveram um modelo numérico de fonte de quantidade de movimento em regime permanente para investigar o escoamento no interior de um tanque misturador. As componentes de velocidade média previstas por este modelo numérico foram comparadas com dados experimentais e com outro modelo numérico que utiliza dados experimentais de LDA (Laser Doppler Anemometry) como condições iniciais. Os resultados tiveram bom acordo com os dados experimentais e os autores concluíram que o modelo de termo fonte é confiável para a simulação do escoamento em tanques misturadores, e produz previsões realísticas do problema.

Mais trade, Patwardhan (2001) [1] desenvolveu um modelo numérico também baseado no modelo de termo fonte para investigar o comportamento do escoamento no interior de um tanque misturador, neste caso, considerando diferentes tipos de impulsores. Algumas variáveis do escoamento foram comparadas com dados experimentais, tendo bom acordo na maioria das localizações de análise, apresentando algumas discrepâncias apenas nas localizações mais próximas do fundo do tanque e na ponta do impulsor.

Já no trabalho de Dhainaut et al. (2006) [4] foi apresentado uma comparação entre o modelo de malha móvel e o modelo de termo fonte em regime transiente, utilizando o software FLUENT, baseado no MVF, e fazendo uma comparação entre dados experimentais com estes dois modelos propostos. Foram medidos componentes de velocidade na direção horizontal afim de comparar os resultados entre experimental e numérico. Na maioria dos casos, os modelos de termo fonte e malha móvel concordaram com os dados experimentais, mostrando algumas discrepâncias quando as velocidades foram medidas diretamente abaixo do impulsor.

O modelo de malha móvel também foi utilizado no trabalho de Mahmud et al. (2006) [3] para avaliar as principais características do escoamento no interior de um tanque misturador, bem como o nível de concordância entre os resultados numéricos e medições experimentais. As componentes do campo de velocidade acima e abaixo do impulsor foram calculadas e comparadas com dados experimentais. Embora existam algumas discrepâncias entre os dados calculados e medidos experimentalmente, as grandezas e os comportamentos dos perfis de velocidade são, em geral, bem reproduzidos pelo modelo CFD.

Segundo Hunag \& Li (2013) [5] apresentaram uma equação para calcular o termo fonte de quantidade de movimento considerando tanto a força propulsora do impulsor quanto o efeito de 
atrito radial entre as lâminas e o fluido. O campo de velocidades próximo ao impulsor e no restante do tanque foram comparados com dados experimentais. Foi feita também uma comparação entre a precisão computacional e o tempo computacional com o modelo de malha móvel e Multiple Rotating Reference Frames - MRF (malha fixa).

Para o presente trabalho, a proposta é a de simular numericamente o princípio de funcionamento de um tanque misturador, utilizando o modelo de malha móvel e de termo fonte, e comparar os resultados obtidos com os resultados apresentados por Huang et al. [5]. Serão feitas também comparações com relação ao tempo e esforço computacional dos dois modelos numéricos. O objetivo é o de validar estes dois modelos e comprovar o ganho de tempo computacional e a simplicidade na construção da geometria e da malha quando utilizado o modelo de termo fonte.

Para a solução das equações de conservação de massa e quantidade de movimento médias no tempo, foi empregado o MVF através do software FLUENT 14.5. A simulação numérica foi realizada considerando um regime de escoamento turbulento, logo, para o fechamento da turbulência foi empregado o modelo $k-\varepsilon$ (RANS - Reynolds Averaged Navier-Stokes).

\section{METODOLOGIA NUMÉRICA}

Foram realizadas duas simulações numéricas, uma com o modelo de malha móvel e outra com o modelo de termo fonte, com o objetivo de analisar o comportamento do escoamento no interior de um tanque misturador. O software proposto para realizar estas simulações é o FLUENT, baseado no Método dos Volumes Finitos [6, 7].

\subsection{Modelo de malha móvel}

No modelo de malha móvel, o tanque é dividido em duas regiões que serão tratadas separadamente, a região do impulsor e a região do tanque (que inclui o fluido, as paredes do tanque, o fundo do tanque e os anteparos). A malha presente na região do impulsor, rotaciona junto do mesmo enquanto a região do tanque permanece estacionária, desta forma, as malhas deslizam através de uma interface cilíndrica. Este método é capaz de capturar com precisão a evolução do escoamento no tempo, mas também o mais computacionalmente exigente.

O domínio computacional criado para simular o princípio de funcionamento do tanque misturador, utilizando o modelo de malha móvel, consiste de um vaso cilíndrico com uma turbina Rushton instalada em um eixo central, e que está a uma determinada distância do fundo do tanque. Quatro anteparos estão espalhados ao redor do tanque com uma distância igual entre eles. Este domínio computacional segue as dimensões do tanque apresentado no estudo de Hunag \& Li (2013) [5], e pode ser observado na Figura 2, com suas respectivas dimensões apresentadas na Tabela 1.

A malha criada para o domínio computacional mostrado na Figura 1, possui 465.516 elementos hexaédricos.
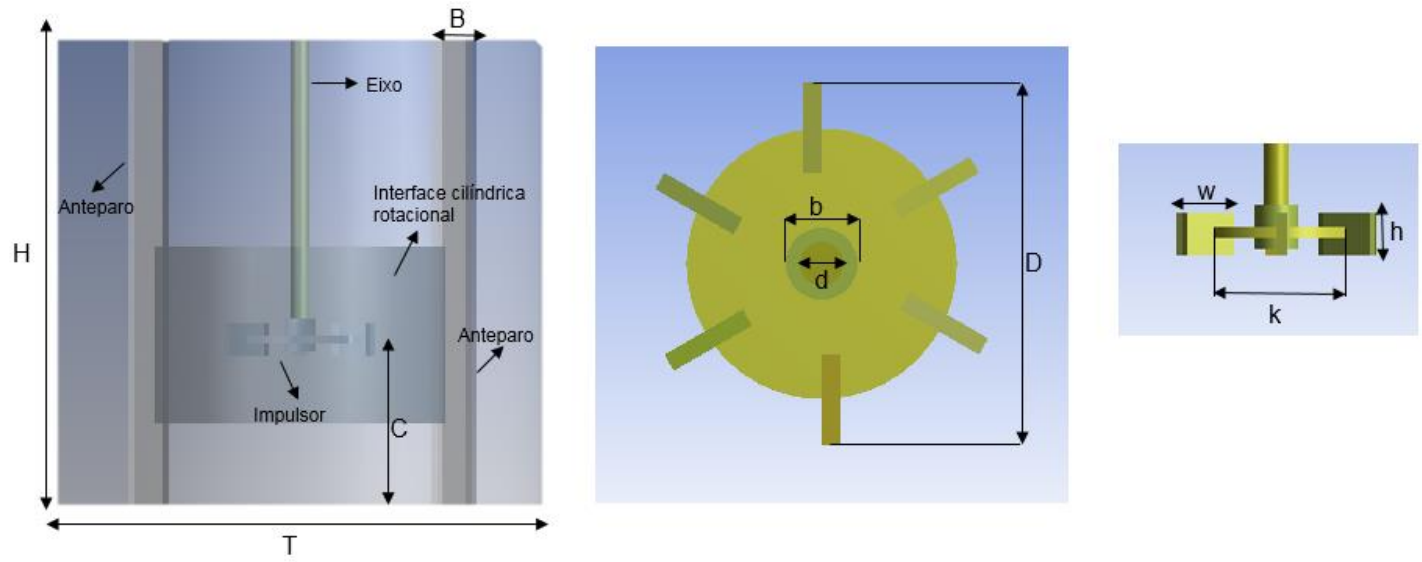

Figura 1: Domínio computacional para o modelo de malha móvel. Fonte: Autores. 
Tabela 1: Dimensões do domínio computacional (m).

\begin{tabular}{cccccccccc}
$\mathbf{T}$ & $\mathbf{H}$ & $\mathbf{C}$ & $\mathbf{B}$ & $\mathbf{D}$ & $\mathbf{b}$ & $\mathbf{d}$ & $\mathbf{w}$ & $\mathbf{k}$ & $\mathbf{h}$ \\
\hline $\mathbf{0 , 3}$ & 0,3 & 0,1 & 0,03 & 0,1 & 0,02 & 0,012 & 0,025 & 0,075 & 0,02 \\
\hline
\end{tabular}

\subsection{Modelo de termo fonte}

Neste modelo, a turbina do tanque é substituída e representada por uma geometria derivada da revolução de uma das lâminas desta turbina, onde o formato se aproxima de um toróide, cujo raio é do tamanho da área atuante se houvesse a turbina. Então, o movimento é descrito a partir de uma fonte de quantidade de movimento, adicionada na equação de conservação de momentum. A malha para este modelo é totalmente fixa, o que auxilia na diminuição do esforço e do tempo computacional. Outro aspecto importante, é que neste caso é possível representar apenas parte do domínio computacional, (considerando o comportamento simétrico do problema), além de realizar a simulação no regime permanente, o que também reduz o esforço e o tempo computacional. O domínio computacional, Figura2, para este modelo seguiu as mesmas dimensões apresentadas na Figura 1, e a malha criada para este domínio possui 1.447.296 elementos hexaédricos. A superfície com velocidade mostrada na Figura 2, bem como o eixo são tratadas como superfícies móveis. $\mathrm{O}$ disco atuador, corresponde à geometria que substitui $\mathrm{o}$ impulsor, e é nele que a condição de termo fonte é adicionada. As duas paredes laterais, incluindo a parede do disco atuador, são tratadas como periódicas. A superfície livre foi tratada como uma tampa plana e rígida, e uma condição de parede deslizante foi dada à superfície do topo. O restante é tratado como parede.

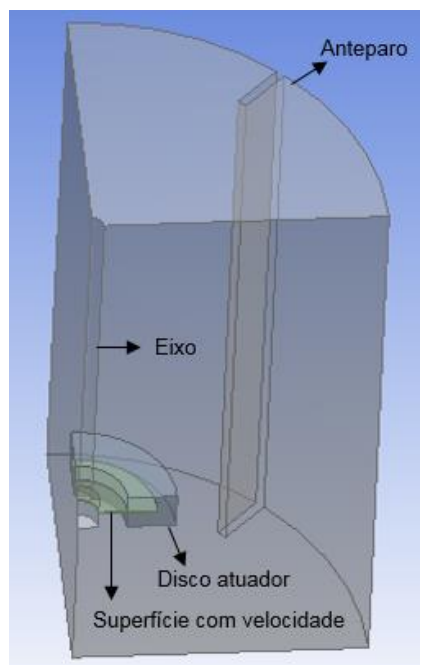

Figura 2: Domínio computacional para o modelo de termo fonte. Fonte: Autores.

A grande diferença entre estes dois modelos é que as simulações envolvendo malha móvel, apesar de serem bastante precisas, são muito mais demoradas, e exigem um esforço computacional maior, já que ocorrem em regime transiente, e possuem uma interface móvel, o que faz com que os cálculos sejam ainda mais demorados e complexos. Por outro lado, o modelo de termo fonte permite que a malha possa ser bem refinada, (já que o esforço computacional, neste caso, está muito mais ligado ao refinamento de malha), fazendo com que sejam alcançados resultados também precisos. Além disso, o regime de escoamento é considerado permanente e a malha é fixa. Outra vantagem com relação ao modelo de termo fonte, é que neste caso, é permitido uma subdivisão da geometria, com relativa flexibilidade na construção da malha, já que não existem as paredes internas do impulsor. Assim, o esforço e o tempo computacional são diminuídos consideravelmente. 


\subsection{Simulação numérica}

Nas duas simulações considerou-se que o tanque estava preenchido com água. No entanto, para o caso com o modelo de termo fonte, a simulação numérica foi realizada considerando apenas $1 / 4$ de todo o domínio computacional. A velocidade de rotação do impulsor para os dois casos foi de $250 \mathrm{RPM}$ gerando $u_{\text {tip }}=1,31 \mathrm{~m} / \mathrm{s}$. Monitores para controlar as componentes de velocidade nas direções axial e radial, foram criados em diferentes alturas, acima e abaixo do impulsor, variando sua localização na direção radial. Os resultados das simulações numéricas com os dois modelos numéricos foram comparados com resultados obtidos no estudo de Huang \& Li (2013) [5]. O tempo e esforço computacional entre estes modelos também foram comparados.

\section{MODELAGEM MATEMÁTICA}

As equações que descrevem o escoamento de um fluido são as equações de conservação de massa e quantidade de movimento, estas também podem ser chamadas de equações de NavierStokes [8].

$$
\begin{gathered}
\nabla u=0 \\
\rho \frac{\partial u}{\partial t}+\rho u . \nabla u=-\nabla p+\mu \nabla^{2} u+f
\end{gathered}
$$

onde $\rho$ é a massa específica, $\mu$ é a viscosidade dinâmica, $u$ é a velocidade, $p$ é a pressão e $f$ é um termo adicional.

No caso do modelo com termo fonte, o termo adicional $f$, presente na Eq. 1, foi determinado a partir das recomendações apresentadas no trabalho de [5]. Para isto, foi criada uma UDF (UserDefined Function) para simular o movimento das lâminas do impulsor se este existisse. A UDF é basicamente uma função definida pelo usuário, em linguagem de programação que pode ser carregada dinamicamente com o solucionador FLUENT com o objetivo de aprimorar os recursos padrão do software [6]. Desta forma, foi possível definir o termo fonte para o regime de escoamento deste problema. O equacionamento utilizado na criação da UDF é mostrado a seguir.

Segundo Huang \& Li (2013) [5] a força exercida pelo impulsor é dada por,

$$
d F=\rho . d S \cdot u\left(u-v_{\theta}\right)
$$

onde $d S$ é um elemento de área na superfície da lâmina, $u$ é a velocidade linear do elemento de área na superfície da lâmina e $v_{\theta}$ é a velocidade tangencial.

Para considerar o efeito do arrasto no movimento do fluido, uma equação de resistência é introduzida para calcular a força de arrasto $d f$ dada como,

$$
d f=\frac{1}{2} \rho \cdot v_{r}^{2} \cdot C_{f} \cdot d S
$$

onde $v_{r}$ é a velocidade radial, $C_{f}$ é o coeficiente de atrito local em função de Reynolds, que é calculado como

onde $R e_{x}$ é dado por,

$$
\begin{gathered}
C_{f}=0,664 \cdot R e_{x}^{-0,5} \\
R e_{x} \leq 5 \times 10^{5} \\
C_{f}=0,0577 \cdot R e_{x}^{-0,2} \\
5 \times 10^{-5}<R e_{x}<1 \times 10^{7}
\end{gathered}
$$

$$
R e_{x}=\frac{\rho v_{r} x}{\mu}
$$

onde $x$ é a distância entre o centro da célula computacional e o centro do eixo de rotação.

Para as duas simulações, foi utilizado o modelo de turbulência $K-\varepsilon$ Standard [8], com esquemas de discretização de primeira ordem para as quantidades turbulentas e de segunda ordem para a continuidade. O método SIMPLE foi utilizado para o acoplamento pressão/velocidade. 


\section{RESULTADOS E DISCUSSÃO}

Nesta seção serão apresentados os resultados obtidos a partir da simulação numérica com o modelo de malha móvel e também com o modelo de termo fonte. Serão apresentados os campos quantitativos e qualitativos de velocidades para os dois modelos simulados. Nos dois casos os resultados foram comparados com dados numéricos encontrados na literatura.

\subsection{Resultados qualitativos}

Para fins de comparação qualitativa, o campo dos vetores de velocidade média para o caso com modelo de malha móvel, Figura 3 (b), e para o caso com modelo de termo fonte, Figura 3 (c), foram plotados e comparados com o campo vetorial apresentado por Hunag \& Li (2013) [5] Figura 3 (a). Este resultado foi obtido através de um plano vertical criado a partir do eixo do impulsor até o ponto médio de dois anteparos do tanque. No entanto, apenas metade do plano é apresentado, já que está sendo considerada a simetria no comportamento do escoamento.

Como pode-se perceber, nos dois casos o escoamento avança na direção radial a partir do impulsor até atingir a parede do tanque, então sua velocidade diminui e o escoamento se separa em duas correntes, para cima e para baixo, após, o escoamento circula pelo tanque até voltar para a região do impulsor repetindo o processo mencionado. Nota-se ainda, que a região onde $z / T>$ 0,4 possui uma recirculação mais suave do que a região $\operatorname{com} z / T<0,3$. Este comportamento ocorre por que o impulsor está mais próximo do fundo do tanque, e isso faz com que a recirculação neste local seja mais evidente. $\mathrm{O}$ mesmo acontece para o caso com o modelo de termo fonte.

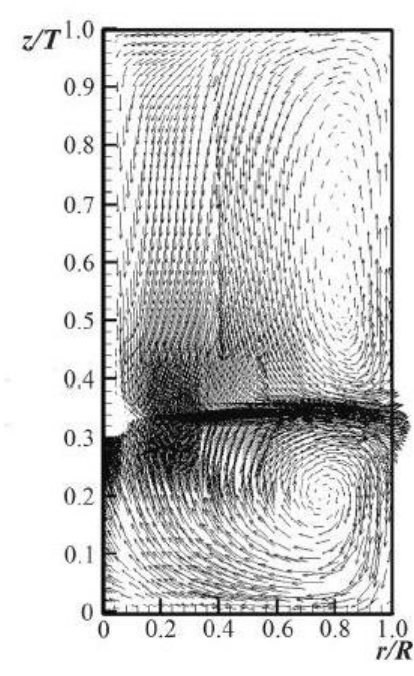

(a)

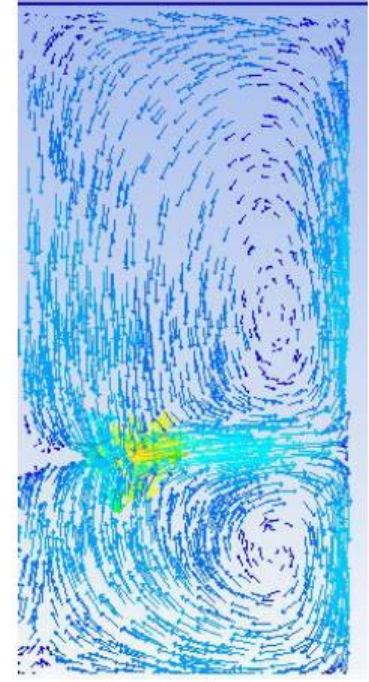

(b)

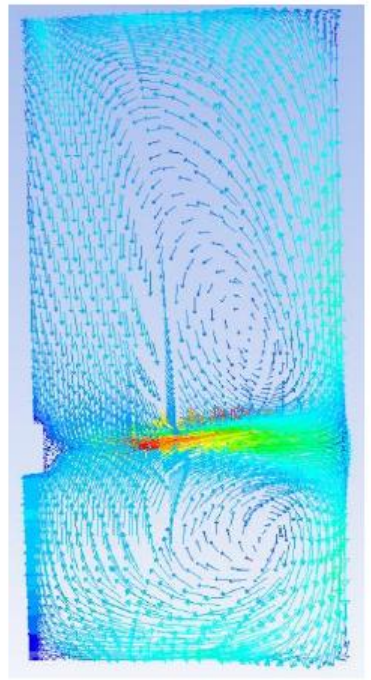

(c)

Figura 3: Campo vetorial. (a) (Huang \& Li, 2013 [5]); (b) modelo de malha móvel; (c) modelo de termo fonte. Fonte: Autores.

\subsection{Resultados quantitativos}

Os resultados obtidos com as simulações numéricas utilizando os modelos de malha móvel e o modelo com termo fonte foram comparados com os resultados mostrados no estudo de Hunag \& Li (2013) [5]. A Figura 4 mostra a velocidade média axial em diferentes alturas com componente radial variável. 


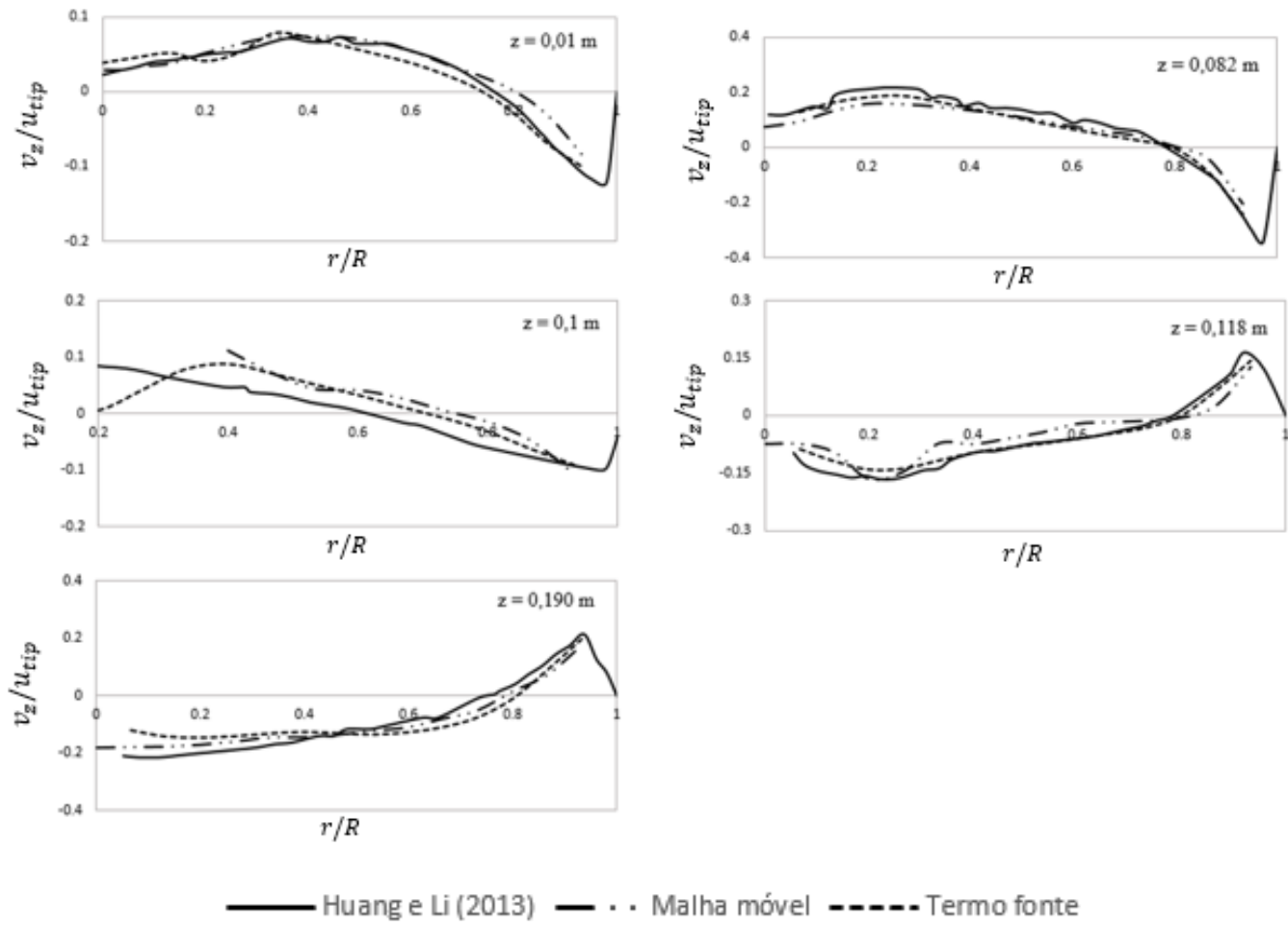

Figura 4: Comparação de resultados para a velocidade axial.

Percebe-se, a partir dos gráficos mostrados na Figura 4, que as velocidades axiais nas duas localizações acima da altura $0,1 \mathrm{~m}$ (acima do impulsor) possuem valores positivos, ao contrário das velocidades abaixo da altura $0,1 \mathrm{~m}$ (abaixo do impulsor) que possuem valores negativos. Essa mudança de sinal nas velocidades é justificada pelo fato de que há uma mudança na direção do escoamento acima e abaixo do impulsor. Essa mudança pode ser também observada nas Figuras. 3 (b) e (c), onde são mostrados os vetores apontando para cima quando estão na parte inferior do impulsor, e apontando para baixo, quando estão na parte superior do impulsor.

A Figura 5 mostra a velocidade média radial também em diferentes alturas e com componente radial variável.

Para as alturas $z=0,01 \mathrm{~m} \mathrm{e} z=0,082 \mathrm{~m}$ (abaixo do impulsor) as velocidades radiais têm um pico máximo entre as razões de 0,6 e 0,8 ainda próximos do impulsor. Depois disso, as velocidades começam a diminuir até alcançar zero à medida que chegam próximo à parede do tanque. Na altura $z=0,1 \mathrm{~m}$ (altura onde se encontra o impulsor) a velocidade radial está inicialmente próxima de zero, já que o fluido está sob a influência das paredes do impulsor. Após, a velocidade radial alcança seu máximo próximo à razão 0,4 do gráfico (na ponta da lâmina), e então, quanto mais próximo da parede menor a velocidade radial. Para as duas alturas acima do impulsor, $z=0,118 \mathrm{~m}$ e $z=0,190 \mathrm{~m}$, ocorre o mesmo comportamento que nas alturas acima do impulsor, isto é, a velocidade radial começa a aumentar, alcança o valor máximo entre as razões de 0,6 e 0,8 e então começa a cair à medida que chega próximo à parede.

Os perfis de velocidade obtidos no presente trabalho seguem a mesma tendência dos resultados apresentados na literatura, com exceção de alguns pontos. Assim, em geral, os resultados apresentados com o modelo de malha móvel e com o modelo de termo fonte, estão próximos dos resultados apresentados no estudo de Hunag \& Li (2013) [5], o que verifica a modelagem desenvolvida no presente trabalho. Dessa forma, acredita-se que os modelos apresentados possam ser utilizados em futuros estudos relacionados a análise de parâmetros geométricos do tanque sobre o comportamento do escoamento. 

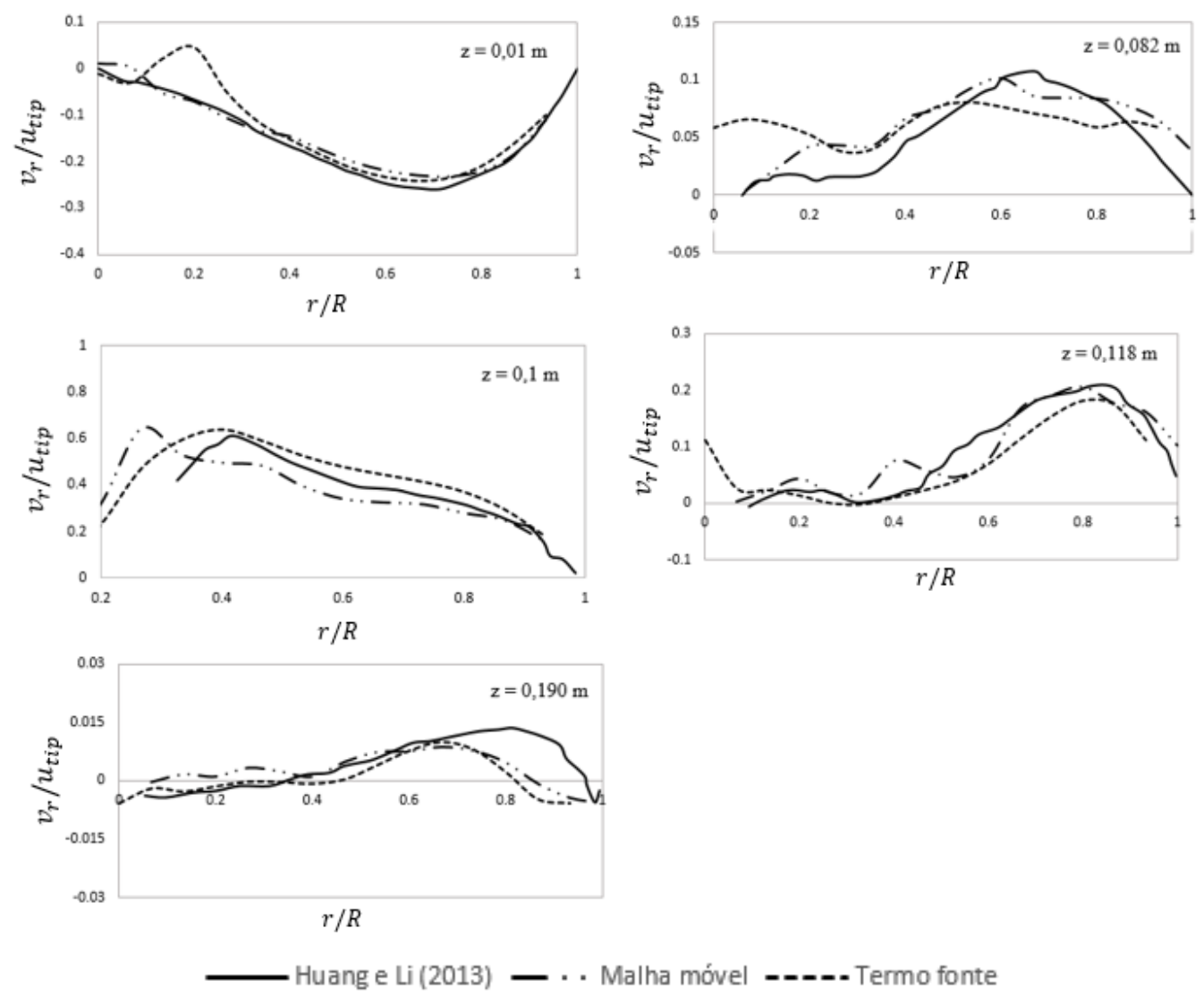

Figura 5: Comparação de resultados para a velocidade radial.

\subsection{Tempo computacional}

As simulações foram realizadas em uma máquina que possui um total de 12 núcleos, processador Intel Core i-7, sistema operacional de 64 Bits e memória RAM de 16 GB. Por questões operacionais, foram utilizados 4 núcleos do total de 12 para simular cada caso. A simulação com malha móvel levou 17 dias para rodar um caso em regime transiente, com 27.000 passos de tempo, com um passo de tempo de $\Delta t=1,0 \times 10^{-3} \mathrm{~s}$, simulando assim, um tempo final de $t=27,0 \mathrm{~s}$. Todos os resultados gerados com os $27 \mathrm{~s}$ de simulação foram utilizados para a montagem da média dos perfis de velocidade.

A simulação com termo fonte, levou 19 horas para simular um caso em regime permanente com um total de 20.000 iterações, ou seja, $4 \%$ do esforço computacional requerido com o modelo de malha móvel.

\section{CONCLUSÃO}

Foi realizada uma comparação entre dois modelos numéricos, malha móvel e termo fonte, para a simulação de um tanque misturador, com o objetivo de simular numericamente o princípio de funcionamento deste, bem como analisar o comportamento do escoamento no interior do mesmo.

Os resultados obtidos foram comparados com resultados encontrados na literatura. Em geral, os dois modelos apresentaram resultados relevantes com relação à previsão do comportamento do escoamento no interior do tanque, bem como, bom acordo com dados encontrados na literatura, podendo ser aplicados em estudos futuros relacionados a análise paramétrica sobre o comportamento do escoamento. A grande diferença entre os dois modelos apresentados, está ligada ao tempo e esforço computacional que cada um deles possui, sendo o modelo de malha móvel excessivamente mais demorado do que o modelo de termo fonte. Assim, pode-se dizer que 
o modelo de malha móvel é também mais exigente computacionalmente do que o modelo de termo fonte.

\section{AGRADECIMENTOS}

O presente trabalho foi realizado com apoio da Coordenação de Aperfeiçoamento de Pessoal de Nível Superior - Brasil (CAPES) - Código de Financiamento 001. Os autores também agradecem ao Programa de Pós-Graduação em Modelagem Computacional (PPGMC - FURG), ao Instituto Federal do Rio Grande do Sul e à ESSS Ltda. O Autor E. D. dos Santos agradece ao CNPq pela bolsa de produtividade em pesquisa (Processo: 306024/2017-9).

\section{REFERÊNCIAS BIBLIOGRÁFICAS}

1. Patwardhan AW. Prediction of Flow Characteristics and Energy Balance for a Variety of Downflow Impellers. Ind Eng Chem Res. 2001;40(17):3806-16, doi: 10.1021/ie000455x.

2. Xu Y, Mcgrath G. CFD Predictions of Stirred-Tank Flows. Chemical Engineering Research \& Design. $4^{\circ}$ ed 1996;471-5.

3. Javed KH, Mahmud T, Zhu JM. Numerical simulation of turbulent batch mixing in a vessel agitated by a Rushton turbine. Chem Eng Process Process Intensif. 2006;45(2):99-112, doi: 10.1016/j.cep.2005.06.006.

4. Dhainaut M, Tetlie P, Bech K. Modeling and Experimental Study of a Stirred Tank Reactor. Int J Chem React Eng [Internet]. 2006 ;3(1). doi: 10.2202/1542-6580.1274

5. Huang W, Li K. CFD Simulation of Flows in Stirred Tank Reactors Through Prediction of Momentum Source. In: Guillen D, organizador. Nuclear Reactor Thermal Hydraulics and Other Applications [Internet]. InTech; 2013. doi: 10.5772/51754.

6. Fluent A. ANSYS FLUENT 12. 0 User's Guide. 2009.

7. Versteeg HK, Malalasekera W. An introduction to computational fluid dynamics: the finite volume method. Nachdr. Harlow: Pearson/Prentice Hall; 2005. 257 p.

8. Szablewski W. B. E. Launder and D. B. Spalding, Mathematical Models of Turbulence. 169 S. m. Abb. London/New York 1972. Academic Press. Preis geb. \$ 7.50. ZAMM - Z Für Angew Math Mech. 1973;53(6):424-424, doi: 10.1002/zamm.19730530619. 